
cultures

Les cahiers de l'Acedle

18-2 | 2021

Didactique des langues \& plurilinguisme(s) : 30 ans de recherches

\title{
Genèse et représentations de répertoires plurilingues
}

Georges lüdi

\section{CpenEdition}

Journals

Édition électronique

URL : https://journals.openedition.org/rdlc/9485

DOI : $10.4000 /$ rdlc. 9485

ISSN : 1958-5772

Éditeur

ACEDLE

Référence électronique

Georges lüdi, "Genèse et représentations de répertoires plurilingues », Recherches en didactique des langues et des cultures [En ligne], 18-2 | 2021, mis en ligne le 30 septembre 2021, consulté le 11 octobre 2021. URL : http://journals.openedition.org/rdlc/9485 ; DOI : https://doi.org/10.4000/rdlc. 9485

Ce document a été généré automatiquement le 11 octobre 2021.

\section{(i) 89

Recherches en didactique des langues et des cultures is licensed under a Creative Commons AttributionNonCommercial-NoDerivatives 4.0 International License 


\title{
Genèse et représentations de répertoires plurilingues
}

\author{
Georges lüdi
}

\section{Introduction}

1 Dans les années 1980, l'équipe de recherche Bâle-Neuchâtel s'était lancée dans une série de recherches sur la dimension linguistique des migrations en rapprochant deux courants de réflexion, largement séparés jusqu'à cette date, qui étaient le plurilinguisme et l'acquisition de langues secondes (voir déjà la version allemande de Lüdi et Py en 1984). Nous nous étions inspirés notamment des travaux de Louise Dabène et de son entourage (voir par exemple, Dabène et al. 1990) et considérions l'acquisition - non guidée - de la langue d'accueil par les migrants comme un cas de bilinguisme émergent (Lüdi, 1984). Progressivement, nous avons adopté une conception socioconstructiviste de l'acquisition concevant la cognition comme partagée et contextuelle (Mondada, 2001) et les structures linguistiques comme temporelles, différées et émergentes (Hopper, 1998 ; Larsen-Freeman \& Cameron, 2008) résultant de, et non pas précédant, la mise en œuvre de l'ensemble du répertoire des acteurs. Nous avons par conséquent développé l'hypothèse que des compétences plurilingues émergent de l'interaction où le statut des marques transcodiques est mutuellement négocié par les participants. Ainsi, devenir plurilingue est compris comme un processus sociocognitif, comme l'expliquent Diane Larsen-Freeman et Lynne Cameron (2008, 158):

Embodied learners soft assemble their language resources interacting with a changing environment. As they do so, their language resources change. Learning is not the taking in of linguistic forms by learners, but the constant (co-)adaptation and enactment of language-using patterns in the service of meaning-making in response to the affordances that emerge in a dynamic communicative situation. ${ }^{1}$

Nous nous concentrerons sur le plurilinguisme individuel conçu comme une des manifestations de la diversité linguistique comme il ressort du champ conceptuel suivant : 
Figure 1 : Les manifestations de la diversité linguistique

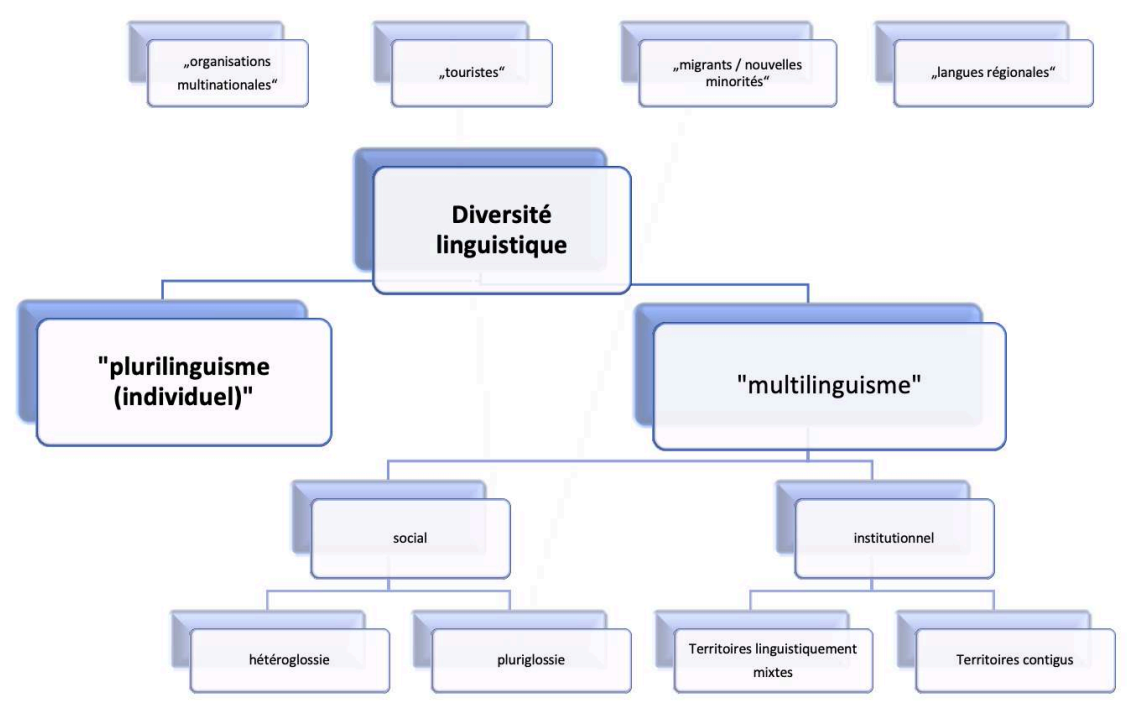

2 L'approche choisie se focalise sur les représentations du plurilinguisme en mettant en exergue ce que nous enseigne le « discours sur » (ce dont nous avons conscience, voire ce que nous pouvons ou voulons dire) en contraste avec ce que l'on peut conclure de l'analyse des "pratiques» (ce que nous faisons / savons, voire ce qui est observable), ceci en fonction du contexte et de la gestion des langues en vigueur (voir Berthoud et al., 2013) :

Figure 2 : Les quatre dimensions du cadre conceptuel de DYLAN et leur mise en relation

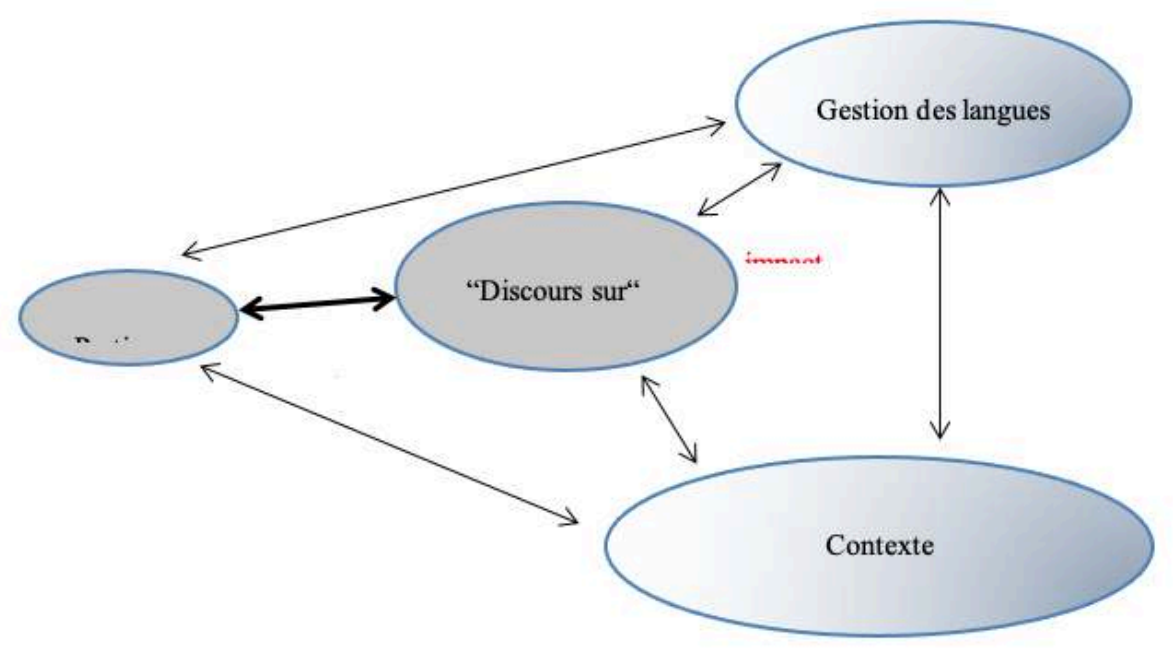

\section{Les représentations}

Quand nous parlons de représentations du plurilinguisme, nous nous focalisons moins sur l'image que s'en font les individus que sur les représentations sociales, à savoir une forme de connaissance collective, socialement élaborée et partagée, concourant à la 
construction d'une réalité commune à un ensemble social (Jodelet, 1984). Les croyances collectives sont en même temps structurées et structurantes par rapport à la communauté qui les supportent et résultent de pratiques discursives complexes (Duchêne, 2005, 34ss.). Nous y avons accès d'une part à travers ce que les acteurs disent, d'autre part à travers l'analyse conversationnelle de leurs comportements.

Dans un premier temps, on constate que ces représentations sont complexes et complémentaires et peuvent être regroupées sous deux titres :

«Je participe à plus d'une communauté linguistique, donc je suis plurilingue.»

«Je mélange, donc je suis plurilingue. »

5 La première représentation se retrouve fréquemment dans le discours des informateurs sur leur emploi des langues avec une forte composante identitaire. On citera ainsi les propos d'un élève d'origine hongroise scolarisé en Autriche cités par Krumm et Jenkins (2001, 89 ; notre traduction) :

Mon cœur est hongrois. Les parties les plus importantes de mon corps sont hongroises. Mon ventre est italien parce que j'aime la cuisine italienne. Mes jambes sont allemandes parce que je vis dans un pays germanophone. Mes bras sont anglais parce que j'aurai besoin de l'anglais pour travailler quand je serai grand.

6 Ou encore les réponses des jeunes migrants citées par Clerc et Cortier (2005) qui justifient leur emploi de la langue d'origine et du français respectivement, en indiquant des raisons affectives et d'appartenance ou, au contraire, utilitaires, mais insistant aussi sur ce que l'on pourrait appeler leur double appartenance :

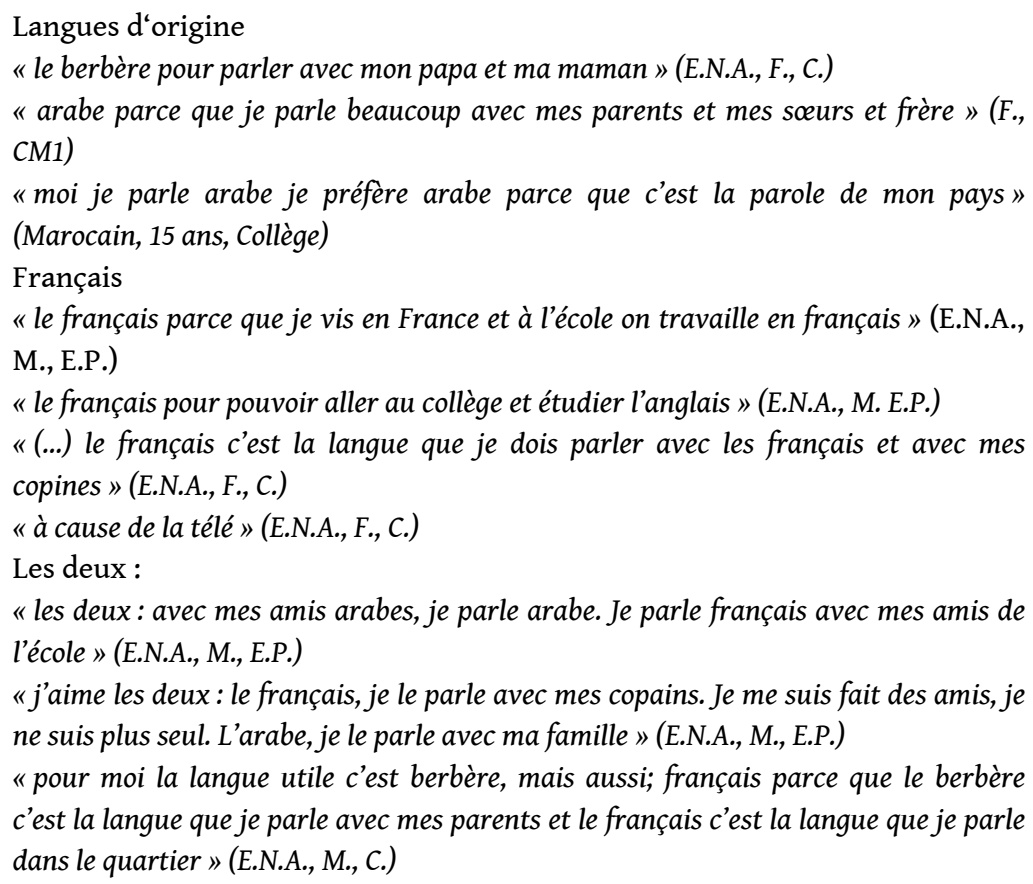

$7 \quad$ Mais une prise de conscience plus prononcée des caractéristiques du plurilinguisme et du «mélange» (parler plurilingue) est aussi observable, comme en témoignent les informatrices de Bouhassane (2007 [cité d'après une présentation PowerPoint en ligne http://www.pedagogie.ac-aix-marseille.fr/jcms/c_167393/de/babel-a-lecoleconference-de-stephanie-clerc-2008]) : 
« $\mathrm{E}$ : beaucoup de langues mélangées est ce que ça existe une personne quand elle parle elle mélange des langues?

Ouafa : oui ça existe

E : ça existe Ouafa

Ouafa : c'est comme ici dehors on mélange le français et l'arabe

$\mathrm{E}:$ ah très bien

Souad: nous aussi chez nous

Ra : moi aussi

$\mathrm{E}:$ alors chez toi vous mélangez quoi Inès?

In : l'arabe français et l'italien

$\mathrm{E}$ : et est-ce qu'on arrive à se comprendre et pourquoi on y arrive?

Bou : moi je parle le français l'italien l'arabe ma mère comprend un peu le

français et l'italien et très bien l'arabe alors on peut se comprendre ».

\section{L'analyse des comportements}

8 Mais c'est surtout au niveau des pratiques que se manifeste la multiplicité des formes de la mise en œuvre de répertoires pluriels. Nous nous limiterons à présenter quatre exemples typiques.

(a) La cruauté des hommes

«P Mh . et cette réalité Ramuz la gé-né-ra-lise dans : le/ dans cet alinéa par une affirmation très forte très dure et précise ...

$\mathrm{E}$ Ah [c'est]. dernière phrase

P Ouais

E Elle ne pensait pas à la seule chose véritable qu'est la cruauté des hommes

P Ouais . des hommes der Menschen? der Männer?

E Des/ der Menschen .

$\mathrm{P} A \mathrm{Ah}$. ce serait intéressant de voir dans une traduction . vous traduire $: \mathrm{z}$. traduisez 'la cruauté des hommes' toute l'expression

E Euh die Grausamheit . der Menschen

$\mathrm{P}$ Mh .. on peut se d'mander . moi j'traduirais quand même par 'die Grausamkeit der Männer' .. eh : mais .à discuter

E Oui (sourit) $\mathrm{mh} \mathrm{mh+.}$

P Pensez-vous que ce soit réel $\neq \ldots$... pensez-vous $(\nexists)$ que $\operatorname{Ramuz}(\neq)$ ait raison $\neq$ de dire cela . que les hommes . de sexe masculin ou nous tous . nous soyons cruels ... »

Nous sommes en situation d'examen oral de bac français langue étrangère en Suisse alémanique. L'enseignante (P) initie un discourse-related code-switching afin d'exploiter le potentiel dénominatif ponctuellement plus nuancé de l'allemand, qui distingue entre homme-Mensch et homme-Mann. L'étudiante (E) entérine l'alternance bien qu'elle contrevienne à la consigne qui prescrit le français comme seule langue acceptée lors de l'examen.

En situation exolingue-plurilingue, les acteurs définissent interactivement le répertoire plurilingue comme un ensemble de ressources, comprenant des éléments de registres et de 'langues' différentes, et mobilisé conjointement pour trouver des réponses locales à des problèmes pratiques.

10 Néanmoins, le français et l'allemand sont conçus comme des langues séparées; la représentation du bilinguisme est " additive " : l'école enseigne des langues étrangères en premier lieu en vue de communiquer dans un mode unilingue avec les natifs 
respectifs (ici: lecture de textes littéraires), mode qui est pour ainsi dire simulé pendant l'examen (et l'enseignement du FLE au lycée).

(b) Ça t'embêtrait

« LN ((parle très vite)) mais j'entends. toi par exemple . chais pas. quand-t si on t'disait d'aller passer à ::: une semaine .. chais pas .. à Genève... ça $t^{\prime}$.. ça t'embêtrait quoi ? Ou bien tu dirais :: oh .. j'va[is] chez ces welschs.. et puis .. comme ça .. parce que nous .. j'entends .. chais pas .. y a .. eh j'en connais beaucoup qui disent : alors là . chez les Suisses allemands .zut quoi + (pause de 6 secondes)

LNN1 mmhm

LNN2 je n'ai pas po.. pas compris du tout

LN ((même débit)) mais . si tu veux . quand tu vas .. chais pas moi .. chais pas ..quand tu :: on te demande .. au fond . si j'te disais maintenant .. eh bien . tu viens une semaine chez moi . qu'est-ce que tu dirais .. oh ben mince . j'vais pas chez les .. ces Suisses romands . quoi . ça t'embêtrait . tu s'rais +

(pause de 8 secondes)

LNN2 (se gratte derrière l'oreille) goulp

LN mais ça .. ça t'embêt'rait . ça s'rait quequ'chose pour toi qui t'ennuierait .

qu'ça t' ... t'as pas envie d'aller le faire

LNN2 (en se tournant vers LNN1) Was heisst ça t'embêtrait ?

LN Keine Lust ... eh

LNN1 s'tuet dir nit ... äh ... passe güet . wettsch ned gärn goh

LNN2 dert abe?

LNN1 ja .

LNN2 (en regardant LNN1) momol. .

(se tourne vers LN) mais oui

LN ça t' .. ça t'plairait .. comme ça tu dirais pas .. eh .. oui . j'vais chez les

Suisses romands . quoi ..

LNN2 non $<$ Langenthal I, $8: 2>$ ».

Dans cet extrait, la représentation sous-jacente est celle du multilinguisme territorial en Suisse. Dans leurs régions respectives, le suisse-allemand et le français dominent largement. Les systèmes éducatifs prévoient des échanges scolaires entre élèves romands et alémaniques. C'est dans ce cadre qu'une jeune fille romande (LN) s'entretient avec deux garçons alémaniques (LNN1 et LNN2) dans une salle de classe du lycée de Langenthal.

11 Les répertoires linguistiques des acteurs sont asymétriques ; la situation est exolingue et très légèrement plurilingue (emploi de bribes d'allemand par LN; meilleure compréhension orale $\mathrm{du}$ français de la part de LNN1). Dans une atmosphère de coopérativité - mais LN ne s'efforce aucunement de simplifier son énoncé ou de parler plus lentement -, LNN2 emploie une formulation transcodique (Was heisst ça t'embêtrait?) pour surmonter un obstacle communicatif, à laquelle les deux destinataires, LN et LNN1, réagissent (Keine Lust / s'tuet dir nit ... äh ... passe güet . wettsch ned gärn goh). Le mode de parler choisi par les interlocuteurs (ou imposé par les responsables de l'échange) est unilingue, le recours à la L1 reste une stratégie compensatoire, mais l'efficacité de cette dernière est favorisée par l'existence d'un espace d'interlocution potentiellement bilingue.

Dans ce sens, les acteurs définissent interactivement leurs répertoires plurilingues comme des ensembles de ressources, comprenant des éléments de registres et de 'langues' différentes, lesquels peuvent être exceptionnellement mobilisés conjointement pour surmonter des problèmes pratiques. 


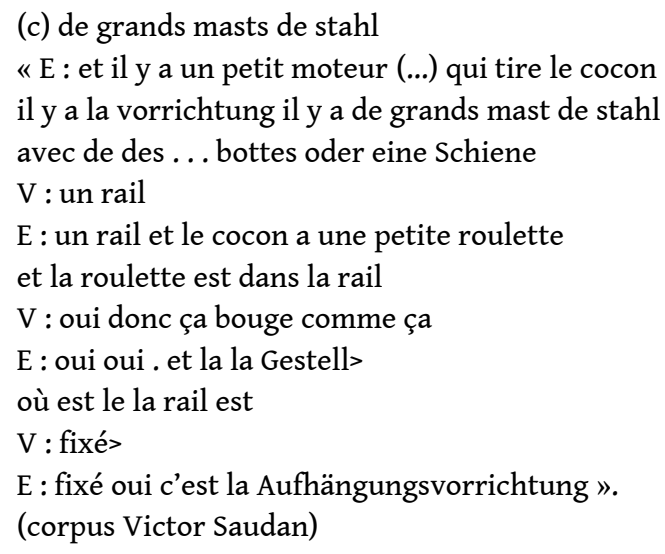

Nous sommes toujours en Suisse, cette fois dans le cadre d'un échange avec un apprenti. E est d'origine alémanique et fait un stage de quelques semaines dans une entreprise romande. Il est en train d'expliquer l'organisation de sa place de travail à son maître d'apprentissage.

13 Le français de E. est très approximatif, en particulier dans le domaine du vocabulaire technique. Ainsi, il insère des unités lexicales allemandes dans des schémas syntaxiques français (de grands masts de stahl). L'interlocuteur francophone réagit sporadiquement. D'une part, il répond à ce qu'il interprète comme un appel à l'aide en proposant des termes français (rail, fixé). Le potentiel acquisitionnel de l'aide fournie est confirmé par le fait que E. répète et réutilise ces termes. Mais souvent V. laisse passer (Vorrichtung, Mast, Stahl, Gestell, Aufhängungsvorrichtung); ce faisant, il signale qu'il a compris et valide ainsi le parler exolingue-bilingue comme mode de communication légitime.

Ainsi, l'expérience plurilingue dans le cadre du stage devient un lieu d'acquisition comme le théorise Larsen-Freeman $(1997,148)$ : "Language grows and organizes itself from the bottom up in an organic way, as do other complex systems."2

(d) y me doile tambem moito la la spalda

« $1 \mathrm{M}$ so hat es doch noch geklappt

$2 \mathrm{P}$ vous parlez français!

$3 \mathrm{M}<$ französisch. > ( ${ }^{\circ}$ oder $\left.^{\circ}\right)$ spanisch?

4 ((manifeste son malaise))

$5 \mathrm{P}$ espanisch. ja

$6 \mathrm{M}$ sie kommen von portugal hab ich gehört ja.

$7 \mathrm{P}^{\circ}$ portugal $^{\circ}$

$8 \mathrm{M} \mathrm{ja}$. (tratamos) en con español.

$9 \mathrm{P}<$ <etabo.>

10 ((très vite; sans doutes «está bem/bom»))

$11 \mathrm{M}$ ok. (..) bueno. (.) puede explicarme eh [tus problemas] síntomas.

$12 \mathrm{P}$ [eh tengo ma!l]e. a la cabeza?

$14 \mathrm{M} \mathrm{mmh}$

15 P eh duo-dolores y e (britisas)?

$16 \mathrm{M} \mathrm{mmh}$

17 P y me doile tambem moito la la spalda.

$18 \mathrm{M}$ la columna! due[le.]

19 P [la ]columna me doi molto! y e: (..) e +<cui> un poco+

20 ((pr. Ital.; touche sa gorge))

$21 \mathrm{M}$ ähä a- +aquít

22 ((touche sa propre gorge))((il note tout ce qu'elle dit))

23 P sí. ho pensato que la gri!pe? +por qu+ la ot[ra ] settimana 


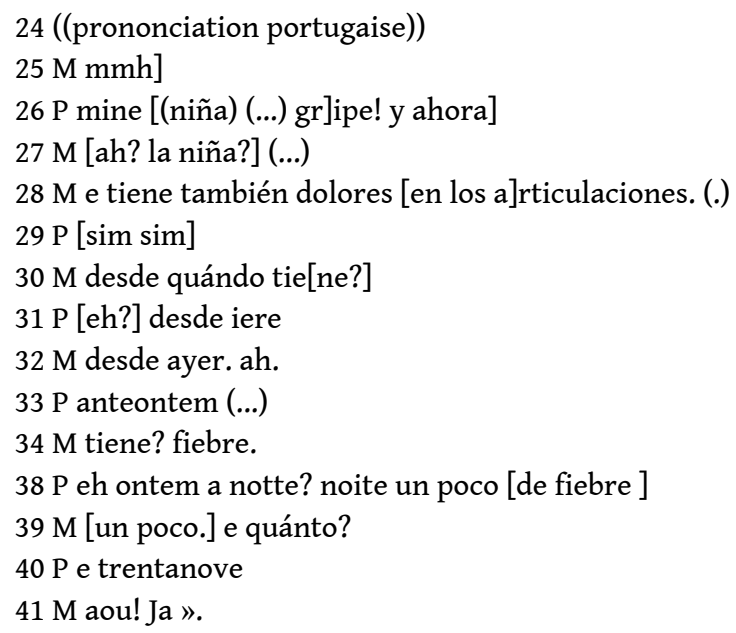

Dans un corpus d'interactions en milieu hospitalier, nous avons trouvé des cas de ce que l'on est tenté d'appeler " pan-roman » où les interlocuteurs (un jeune médecin suisse $\mathrm{M}$ et une malade d'origine portugaise $\mathrm{P}$ ) bricolent des énoncés en situation exolingue extrême. Le médecin et la patiente ne partagent aucune langue commune. Dans les premiers tours de parole, les ressources possibles (allemand, espagnol ou portugais) ou rejetées (français) sont déployées (1. 2-4). M. suggère de choisir l'espagnol (1. 7), choix que P. accepte, mais en portugais (1. 8). En réalité, l'« espagnol » parlé par P. s'avère être un mélange d'espagnol, de portugais et d'italien. $M$. semble assez bien comprendre ce mélange. Pourtant, malgré le travail lexical commun (me doile tambem moito la la spalda reformulé par M. comme la columna duele et de nouveau par P. comme la columna me doi molto [1.14-16]), l'intercompréhension reste approximative, ce qui force les interlocuteurs à faire appel à des gestes déictiques (1. 16-17). Ce faisant, ils se limitent à ce qui est essentiel pour le diagnostic du médecin et négligent des insécurités lexicales mineures (iere 嵁 ayer - anteontem).

En mobilisant ainsi leurs ressources plurielles, M. et P. répondent à la question de recherche formulée par Pennycook: "In what ways do people draw on language resources, features, elements, styles as they engage in translingual, polylingual, metrolingual language practices? ${ }^{3} \gg{ }^{4}$ Ces instances de parler exolingue, qui défient les normes du parler plurilingue usuel, représentent une forme de «bricolage créatif » au sens de Lévi-Strauss (1962: 27) :

la règle de son enjeu est de toujours s'arranger avec les 'moyens du bord', c'est-àdire un ensemble à chaque instant fini d'outils et de matériaux, hétéroclites au surplus, parce que la composition de l'ensemble n'est pas en rapport avec le projet du moment, ni d'ailleurs avec aucun projet particulier, mais est le résultat contingent de toutes les occasions qui se sont présentées de renouveler ou d'enrichir le stock, ou de l'entretenir avec les résidus de constructions et de destructions antérieures.

\section{En guise de bilan}

Dans un premier temps, nos exemples semblent confirmer une vision traditionnelle du bi-/plurilinguisme, qui établit une distinction entre les modes unilingue et bi-/ plurilingue de la mise en œuvre de ressources plurielles (voir Grosjean, 1985). 
Figure 3 : La mise en œuvre d'un répertoire bilingue

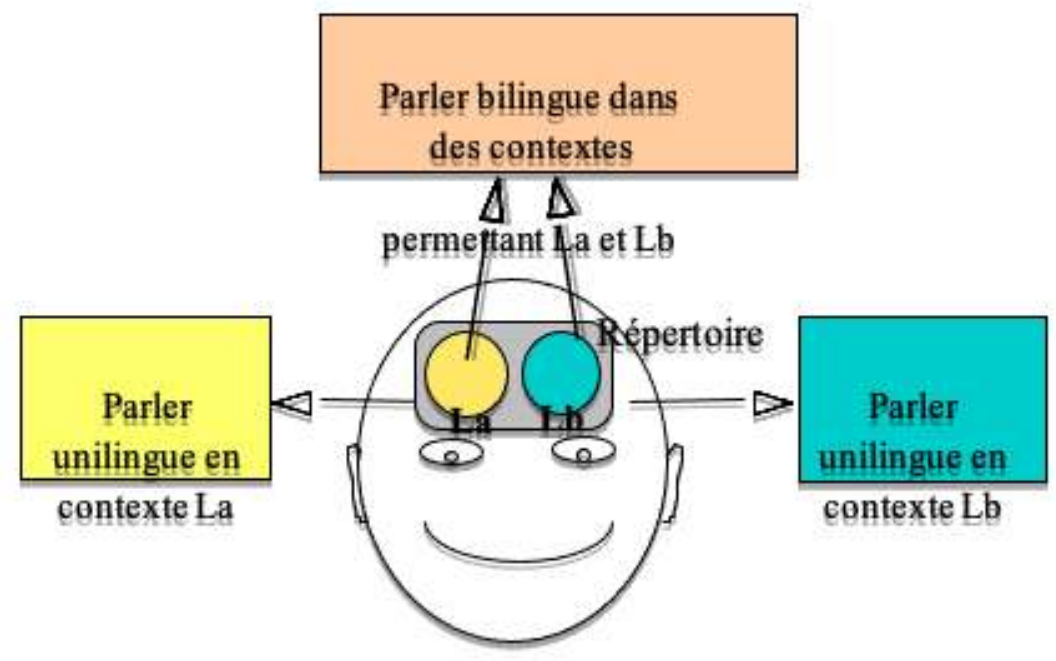

Mais ce modèle n'explique que très partiellement nos observations et exige un cadre épistémologique nouveau. Un premier élargissement tiendra compte de différents degrés de plurilinguisme et d'exolinguisme tels qu'ils sont perçus par les acteurs, c'està-dire de leur catégorisation de la situation dans un système de coordonnées à deux axes :

Figure 4 : Degrés d'exolinguisme-plurilinguisme

\section{exolingue}

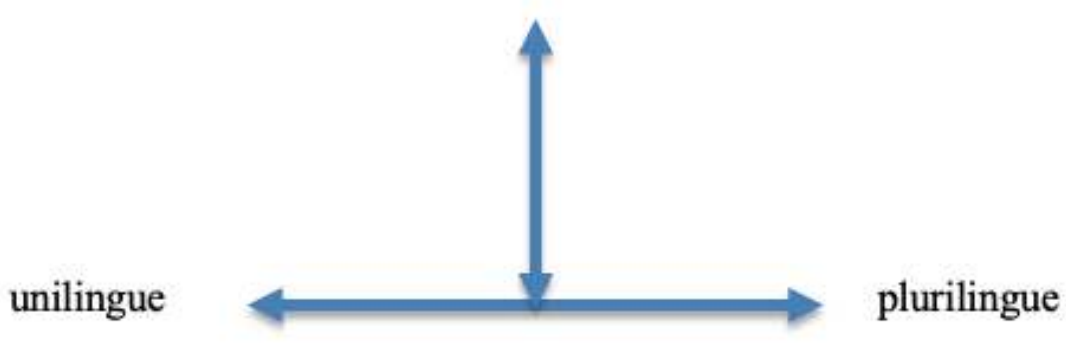

\section{endolingue}

Deuxièmement, de nombreuses représentations - de spécialistes aussi bien que de personnes ordinaires - reposent sur des modèles «additionnistes" des répertoires pluriels comprenant des systèmes linguistiques autonomes, aux frontières nettes ( langues », « compétences »), qui renvoient à l'idée de langues standard aux normes stables, acquises et mises en œuvre séparément, selon le principe des communautés 
linguistiques homoglossiques, par un sujet cognitif autonome; et selon une tradition linguistique bien connue, la priorité appartient à la langue par rapport à la parole.

Cette représentation n'est pas fausse dans la mesure où elle se retrouve partout, mais elle est de plus en plus souvent complétée par un modèle " intégratif ", qui s'appuie sur une conception fonctionnelle du plurilinguisme et sur l'idée que les activités humaines et la cognition sont contextuelles et interactionnelles. Un ensemble de compétences, entre parfaites et très partielles, fonctionne comme un tout intégré (Cook, 2008). Dans cette perspective, la notion de "compétence" est souvent remplacée par celle de " répertoire ", défini comme un ensemble de « ressources » verbales et non-verbales, conjointement mobilisées par les acteurs afin de trouver des solutions locales à des problèmes pratiques. La «langue » y est considérée comme émergente de l'usage (du «languaging »); et les frontières nettes entre les variétés s'estompent (Lüdi \& Py, 2009). C'est bien ce que nous avons observé dans les instances de " pan-roman » citées.

Nous rejoignons ainsi Otsuji et Pennycook (2010: 244), qui se proposaient de démystifier (démythologiser) d'une part la représentation de " mélange des langues » le long de la ligne de faille du bilinguisme, d'autre part la notion d'hybridité.

On postulera par conséquent une "linguistique du plurilinguisme » dans le cadre de laquelle des formes de mélange, de parler plurilingue et d'autres traces de la mise en œuvre de compétences plurilingues telles que nous les avons observées occupent non pas un rôle marginal, mais font partie du cœur même des modèles linguistiques.

\section{BIBLIOGRAPHIE}

Berthoud, A.-C., Grin, F. \& Lüdi, G. (2013). Exploring the Dynamics of Multilingualism. Results from the DYLAN Project. Amsterdam, John Benjamins.

Bouhassane, F.(2007). «L'Eveil aux langues au service de la compétence plurilingue des élèves nouvellement arrivés en France ", Mémoire de Master 2, Université d'Avignon (CLA, Collège P. Eluard, Bollène)

Clerc, S. \& Cortier, C. (2006). « Représentations des langues et des pratiques langagières chez des élèves plurilingues ». 8e Congrès ALA, Plurilinguisme et conscience linguistique : quelles articulations ?, 2-5 juillet 2006, Le Mans.

Cook, V. (2008). Second Language Learning and Language Teaching. London: Arnold.

Dabène, L. \& Billiez, J. (1990). Langues et cultures des populations migrantes, LIDIL, 2. Grenoble, Université Stendhal.

Duchène, A. (2005). Protéger les minorités linguistiques au sein des Nations Unies? Discours, idéologie et production de savoir. Université de Bâle, thèse de doctorat dactylographiée.

Grosjean, F. (1985). «The Bilingual as a Competent but Specific Speaker-hearer. » Journal of Multilingual and Multicultural Development, 6, 467-477. 
Grosjean, F. (2001). « The Bilingual's Language Modes », In Ellen Bialystok (ed.) : Language Processing in the Bilingual. Oxford, Blackwell, 1-25.

Hopper, P. (1998). « Emergent Grammar », In Tomasello, M. (ed.) : The new psychology of language. Mahwah, NJ, Lawrence Erlbaum, 155-175.

Jodelet, D. (1984). « Représentations sociales : phénomènes, concept et théorie », In Moscovici, Serge (éd.) : Psychologie sociale. Paris, PUF, 357-378.

Krumm, H-J. \& Jenkins, E-M. (2001). Kinder und ihre Sprachen - lebendige Mehrsprachigkeit. Wien, Eviva.

Larsen-Freeman, D.\& Lynne C. (2008).Complex Systems and Applied Linguistics. Oxford, Oxford University Press.

Lévi-Strauss, C. (1962). La pensée sauvage. Paris :Plon.

Lüdi, G.(1984). « L'acquisition d'une langue seconde par des migrants: naissance d'un bilinguisme", In Bernard Py, (ed.). Acquisition d'une langue étrangère III, Encrages, Paris/Neuchâtel, 275-281.

Lüdi, G. \& Py, B. (1984). Zweisprachig durch Migration. Einführung in die Erforschung der Mehrsprachigkeit am Beispiel zweier Zuwanderergruppen in Neuenburg (Schweiz). Tübingen, Niemeyer. Lüdi, G.\& Py, B.(2009). « To Be or Not to Be ... a Plurilingual Speaker », International Journal of Multilingualism, 6(2), 154-67.

Lüdi, G. \& Py, B. (2013). Etre bilingue. 4e édition ajoutée d'une postface. Berne. Francfort -s. Main/ New York, Lang.

Mondada, L. (2001). « Pour une linguistique interactionnelle », Marges linguistiques 1, 142-162.

Otsuji, E. \& Pennycook, A. (2010). « Metrolingualism: fixity, fluidity and language in flux », International Journal of Multilingualism, 7: 3, 240-254.

\section{ANNEXES}

\section{Conventions de transcription}

Transcription orthographique (mais sans les majuscules et les signes de ponctuation de l'écrit).

Les transcripteurs ne corrigent en principe pas la langue des locuteurs. 


\begin{tabular}{|c|c|c|c|c|}
\hline & & lène & Conventions & Spécifications et exemples \\
\hline 1 & $\begin{array}{l}1 \mathrm{~b} \\
1 \mathrm{c}\end{array}$ & $\begin{array}{l}\text { Séquentialité } \\
\text { Chevauchements } \\
\text { Enchaînement immédiat } \\
\text { Continuation du tour du } \\
\text { locuteur sur plusieurs lignes } \\
\text { lorsqu'un chevauchement ou un } \\
\text { commentaire est intercalé }\end{array}$ & $\begin{array}{l}{[]} \\
= \\
\&\end{array}$ & $\begin{array}{l}\text { Début et (si possible) fin du } \\
\text { chevauchement sont indiqués } \\
\text { il a à peine fini= } \\
\text { =elle commence } \\
\begin{array}{l}\text { Placé à la fin de la ligne et au début de la } \\
\text { suivante }\end{array}\end{array}$ \\
\hline 2 & $\begin{array}{ll}2 \\
2 \mathrm{a}\end{array}$ & $\begin{array}{l}\text { Silences, pauses } \\
\text { Pause courte } \\
\text { Pauses longues } \\
\text { Pauses d'une seconde et plus } \\
\end{array}$ & $\begin{array}{l}. \\
. . \quad \cdots \\
(1),(2),(3)\end{array}$ & $\begin{array}{l}\text { Environ } 1 / 3 \text { de seconde } \\
\text { Environ } 2 / 3 \text { de seconde }\end{array}$ \\
\hline 3 & $\begin{array}{ll}3 \\
3 \mathrm{a}\end{array}$ & $\begin{array}{l}\text { Indices segmentaux } \\
\text { Troncation }\end{array}$ & $(10,2,2,0)$ & from- fromage \\
\hline 4 & $\begin{array}{l}4 \mathrm{~b} \\
4 \mathrm{~b} \\
4 \mathrm{c} \\
4 \mathrm{~d} \\
4 \mathrm{e} \\
4 \mathrm{f} \\
4 \mathrm{~g}\end{array}$ & $\begin{array}{l}\text { Indices suprasegmentaux } \\
\text { Allongement } \\
\text { Intonation montante } \\
\text { Intonation descendante } \\
\text { Emphase } \\
\text { Accélération du débit } \\
\text { Ralentissement du débit } \\
\text { Voix plus forte } \\
\text { Voix moins forte } \\
\end{array}$ & $\begin{array}{l}: \\
? \\
\vdots \\
\text { >plus vite< } \\
\text { <plus lent> } \\
\text { FORTement } \\
{ }^{\circ} \text { doucement }\end{array}$ & $\begin{array}{l}\text { Les allongements sont notés après la } \\
\text { syllabe concernée: il: y en a beau:coup } \\
\text { Notation après le mot } \\
\text { Notation après le mot } \\
\text { très! bien } \\
\text { En majuscules }\end{array}$ \\
\hline 5 & $\begin{array}{l}5 \\
5 \mathrm{a}\end{array}$ & $\begin{array}{l}\text { Aspiration-expiration } \\
\text { Respiration } \\
\text { Rire } \\
\end{array}$ & $\begin{array}{l}\text { ((soupire)) } \\
\text { (h) }\end{array}$ & $\begin{array}{l}\text { Commentaire entre double parenthèses à } \\
\text { l'intérieur du tour de parole }\end{array}$ \\
\hline 6 & $\begin{array}{l}6 \\
6 \mathrm{a} \\
6 \mathrm{~b} \\
6 \mathrm{c}\end{array}$ & $\begin{array}{l}\text { Doutes de transcription } \\
\text { Hésitations } \\
\text { Alternatives } \\
\text { Segment incompréhensible }\end{array}$ & $\begin{array}{l}\text { (segment pas sûr) } \\
\text { (parce que;car ce que) } \\
\text { (xxx) }\end{array}$ & \\
\hline 7 & 7 & Commentaires & $\begin{array}{l}\text { +segment concerné }+ \\
((\text { commentaire }))\end{array}$ & $\begin{array}{l}\text { Commentaire entre double parenthèses } \\
\text { sur une seconde ligne de transcription }\end{array}$ \\
\hline 8 & 8 & Principes de lectures & & $\begin{array}{l}\text { Les participants qui prennent la parole } \\
\text { sont indiqués à gauche du tour de parole }\end{array}$ \\
\hline
\end{tabular}

\section{NOTES}

1. «Les apprenants incarnés assemblent doucement leurs ressources linguistiques en interagissant avec un environnement en mutation. Ce faisant, leurs ressources linguistiques changent. L'apprentissage n'est pas l'intériorisatiom des formes linguistiques par les apprenants, mais la (co-) adaptation et la mise en œuvre constantes de schémas d'utilisation de la langue au service de la création de sens en réponse aux défis qui émergent dans une situation communicative dynamique " (Notre traduction).

2. «La langue se développe et s'organise de bas en haut de manière organique, comme le font d'autres systèmes complexes. » (Notre traduction).

3. « De quelle manière les gens s'appuient-ils sur les ressources linguistiques, les caractéristiques, les éléments, les styles lorsqu'ils s'engagent dans des pratiques langagières translinguistiquess, polylingues, métrolinguistiques? » (Notre traduction).'

4. La citation provient d'une présentation PowerPoint d'Alastair Pennycook consultée le 20 mars 2010 sur le site www.wesleycollege.net/Our-Community/Wesley-College-Institute/PublicEducation/Global-Language-Convention/Presentations/ /media/Files/

Wesley\%2520College\%2520Institute/Global\%252

oLanguage\%2520Convention/

Alastair\%2520Pennycook.ashx. Le lien n'est plus accessible. 


\section{RÉSUMÉS}

À la suite de recherches sur le bilinguisme émergent de migrants suite à leur l'acquisition non guidée de la langue d'accueil, l'équipe de recherche Bâle-Neuchâtel commença à mettre en doute les modèles traditionnels, additifs, du bi-/plurilinguisme et à partager une conception intégrative de la compétence plurilingue, définissant le répertoire plurilingue comme un ensemble de ressources, comprenant des éléments de registres et de langues différentes entre lesquelles les frontières sont floues. Il est vrai que les conceptions additives restent bien enracinées dans les savoirs quotidiens populaires. Dans cette contribution, nous abordons la question de savoir comment les acteurs plurilingues eux-mêmes conçoivent cette tension en analysant leur discours, mais aussi et surtout les différentes formes de mise en œuvre de leurs répertoires pluriels dans ce qu'il est convenu d'appeler le « mode plurilingue ».

Following research on the emerging bilingualism of migrants in the course of their unguided acquisition of the host language, the Basel-Neuchatel research team began to question the traditional, additive models of bi- / plurilingualism. and to share an integrative conception of plurilingual competence, defining the plurilingual repertoire as a set of resources, comprising elements of different registers and languages between which the boundaries are blurred. It is true that the additive conceptions remain well rooted in people's everyday knowledge. In this paper, we address the question of knowing how the plurilingual actors themselves conceive of this tension by analyzing their discourse, but also and above all the different forms of implementation of their plural repertoires in what it is appropriate to call the 'plurilingual mode'.

\section{INDEX}

Mots-clés : compétence plurilingue, répertoire plurilingue, conception intégrative, savoirs quotidiens

Keywords : plurilingual competence, plurilingual repertoire, integrative conception, everyday knowledge

\section{AUTEUR}

\section{GEORGES LÜDI}

Université de Bâle

Georges LÜDI a enseigné la linguistique générale à l'Université de Neuchâtel et la linguistique française à l'Université de Bâle où il a été doyen de la Faculté des lettres. Il a dirigé de nombreux projets de recherche en sociolinguistique et linguistique appliquée. Ses travaux ont porté principalement sur le plurilinguisme et les langues en contact, l'acquisition des langues étrangères ainsi que sur la politique linguistique éducationnelle. georges.luedi[at]unibas.ch 